Check for updates

Cite this: Phys. Chem. Chem. Phys., 2020, 22, 2590

DOI: 10.1039/d0cp90011g

\section{Correction: Lithium dendrite growth mechanisms in polymer electrolytes and prevention strategies}

\author{
Pallab Barai, ${ }^{a}$ Kenneth Higa ${ }^{b}$ and Venkat Srinivasan*a \\ Correction for 'Lithium dendrite growth mechanisms in polymer electrolytes and prevention strategies' \\ by Pallab Barai et al., Phys. Chem. Chem. Phys., 2017, 19, 20493-20505.
}

rsc.li/pccp

The authors would like to point out an error in one of the equations. The overpotential ( $\eta$ ) used in eqn (2) of the main manuscript has been defined as $\eta=\phi_{\mathrm{s}}-\phi_{\mathrm{e}}-U_{\mathrm{Li}}$ (see left column of the fourth page). Instead the overpotential term should be, $\eta=\phi_{\mathrm{s}}-\phi_{\mathrm{e}}-$ $U_{\mathrm{Li}}+\left(\Delta \mu_{\mathrm{e}^{-}} / F\right){ }^{1,2}$ Here, $\phi_{\mathrm{s}}$ is the solid phase potential, $\phi_{\mathrm{e}}$ is the electrolyte potential, $U_{\mathrm{Li}}$ is the open circuit potential of lithium metal, $F$ indicates the Faraday constant, and $\Delta \mu_{\mathrm{e}^{-}}$is the change in electrochemical potential due to the mechanical stress induced effects. The form of eqn (2) shown in the published version of the manuscript takes into account the impact of mechanical stress induced change in electrochemical potential $\left(\Delta \mu_{\mathrm{e}^{-}}\right)$in the pre-exponential factor, but the effect of $\Delta \mu_{\mathrm{e}^{-}}$was erroneously neglected in the overpotential $(\eta)$ term, which appears inside the exponential.

The correction of the overpotential term does not change the qualitative results shown in the paper; however, it allows better quantitative predictions. To demonstrate how this correction in the overpotential term of the Butler-Volmer equation affects some of the results, we recreate the two key figures in the manuscript, namely, Fig. 4 and 6, with the updated form of overpotential ( $\eta$ ). The modified figures are shown below. It is evident from the corrected figures that the relative magnitude of current density at the peak and the valley change due to incorporation of the extra term into the overpotential expression. However, the overall trend did

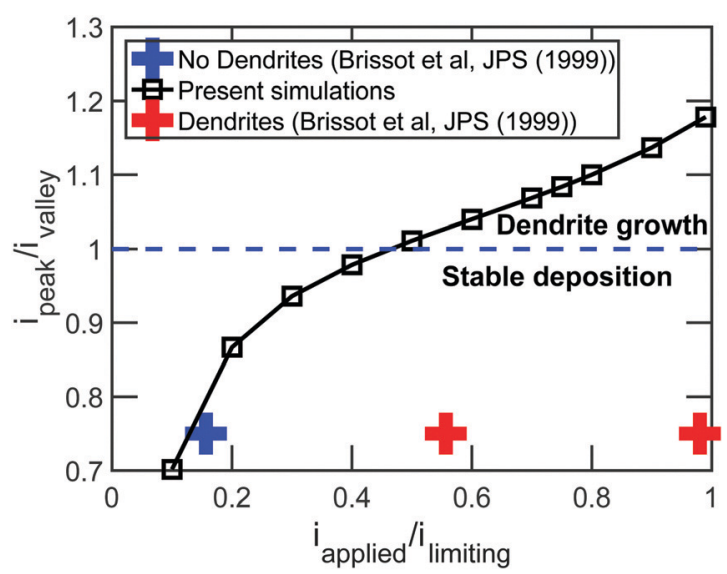

Fig. 4 (Corrected) The ratio of current at the peak over that at the valley with respect to the applied current has been plotted for lithium deposition with PEO polymer-based electrolytes. Increasing the applied current encourages dendrite growth. The blue horizontal line indicates the barrier below which stable deposition of lithium occurs, and above which dendrites can grow. For PEO-based electrolytes, if the applied current is greater than $45 \%$ of the limiting current, present simulations predict that dendrites can grow. From the experimental data, ratio between applied current over limiting current can be obtained (the data points along the $x$-axis). Even though the exact ratio of current at the peak over that at the valley is not known, the fact whether dendrite growth occurs or not, can be extracted from the experimental results. The critical current density predicted in the present research qualitatively correlates with the experimental observations.

\footnotetext{
${ }^{a}$ Argonne National Laboratory, Lemont, Illinois 60439, USA. E-mail: vsrinivasan@anl.gov

${ }^{b}$ Lawrence Berkeley National Laboratory, Berkeley, California 94720, USA
} 


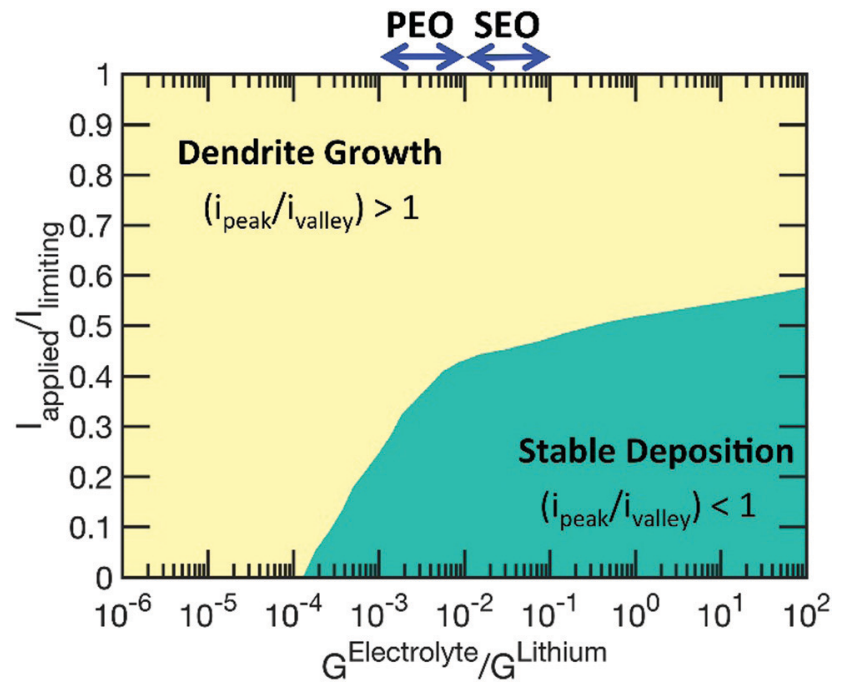

Fig. 6 (Corrected) A phase map of the applied current with respect to the shear modulus of the polymer electrolyte phase. This map indicates that by increasing the elastic modulus of the electrolyte it may be possible to operate the battery at higher currents without the formation of dendrites. Elastic properties of poly(ethylene oxide) (PEO) and poly(styrene ethylene oxide) (SEO) have been adopted from Mullin et al. ${ }^{3}$

not change significantly. Such as, enhanced dendrite growth at higher current densities is still evident from the corrected version of Fig. 4. Similarly, it can still be concluded from the modified Fig. 6 that high modulus electrolytes demonstrate improved suppression of the dendritic protrusion.

We suggest that researchers use the corrected equations in further studies.

The Royal Society of Chemistry apologises for these errors and any consequent inconvenience to authors and readers.

\section{References}

1 C. Monroe and J. Newman, J. Electrochem. Soc., 2004, 151, A880-A886.

2 C. Monroe and J. Newman, J. Electrochem. Soc., 2005, 152, A396-A404.

3 S. Mullin, A. Panday, N. P. Balsara, M. Singh, H. B. Eitouni and E. D. Gomez, US Pat., US 2009/0104523 A1, 2009. 